\title{
Survey on WSN Routing Protocols
}

\author{
Jaspinder Kaur \\ M. Tech Student \\ CTIEMT \\ Shahpur, Jalandhar, India
}

\author{
Taranvir Kaur \\ Assistant Professor \\ Computer science \\ Department, CTIEMT \\ Shahpur, Jalandhar, India
}

\author{
Kanchan Kaushal \\ M. Tech Student \\ CTIEMT \\ Shahpur, Jalandhar, India
}

\begin{abstract}
Recent advances in wireless sensor networks (WSN) over the last two decades has an more interest in the potential use in applications like combat field, security surveillance, border protection, disaster management. Moreover, researchers have termed the 21 st century as the "Sensor Decade" Sensor nodes are remotely deployed in large numbers and expected to operate autonomously in harsh environments [3]. Now-a-days routing protocols are acting as designed, where essential designing issue is energy. In this paper, the survey on current routing protocols for sensor networks along with their classification and approaches is done. Each routing protocol is described under the appropriate category followed by possible future research areas.
\end{abstract}

\section{General Terms}

Greedy Algorithm, micro-electro-mechanical system, WSN, Routing Protocols, energy efficiency, Sensors, Clustering.

\section{Keywords}

WSN, Routing Protocols, nodes, energy.

\section{INTRODUCTION}

Late advances in micro-electro-mechanical system and low power and exceedingly incorporated digital electronics have prompted the improvement of micro-sensors. WSN is a developing data innovative progression in micro- electronics and network communications. It structures an intelligent network application system comprising of a huge number of tiny energy constrained and low cost nodes. Sensor nodes self-organized sense, process and communicate by means of the radio medium shaping a self-organized network design. Ordinarily, sensors report sensed information to an outer base station for further processing. WSN can be characterized as a network of devices, known as nodes, which can sense the Environment and convey the data gathering from the monitored field (e.g. a region of volume) through wireless connections.

Routing in sensor systems is extremely difficult because of a few qualities that recognize them from contemporary communication and wireless ad hoc networks. Above all else, it is unrealistic to assemble a global addressing scheme for the arrangement of sheer number of sensor nodes. Hence, traditional IP-based protocols cannot be connected to sensor network. Second, in contrary to typical communication networks practically all applications of sensor systems oblige the stream of sensed information from various areas (sources) to a particular sink. Third, produced information traffic has significant redundancy in it since different sensors may create same information inside the vicinity of a phenomenon. Such repetition needs to be misused by the routing protocols to improve energy and bandwidth utilization. Fourth, sensor nodes are tightly constrained [4] regarding transmission power, on-board energy, and capacity and thus require careful resource management.

Because of such contrasts, a lot of people new algorithms have been proposed for the issue of routing data in sensor networks. These routing mechanism has considered [2] the attributes of sensor nodes alongside the application and building design prerequisites. Very nearly the majority of the routing protocols can be delegated data-centric, hierarchical or location-based although $[2,3,7]$ there are few distinct ones based on network flow or quality of service (QoS) awareness.

Data-centric protocols are query based and depend with respect to the naming of desired information, which helps in disposing of numerous excess transmissions. Hierarchical protocols focus on clustering the nodes so cluster heads can do some collection and reduction of data in order to save energy. Location based protocols using the position data to relay the information to the desired regions instead of the entire system.

\section{ROUTING PROTOCOLS IN WIRELESS SENSOR NETWORKS}

The network layer handles routing of information over the system from the source to the goal. WSNs Routing protocols varies from customary routing protocols. Routing protocols have an unlimited degree in examination range when actualized in WSN. These routing protocols can be separated on the premise of system structure directing ways created, and system operations and as an initiator of correspondences. Fig 1 demonstrates the scientific categorization of routing protocols which are further sub-isolated into subcategories and accommodating in planning of system protocol. In this area, the routing protocols for WSN are examined.

\subsection{Network Structure based Routing Protocols}

Routing Protocols have system base protocols which are ordered into three sorts and all are carries on in an unexpected way. These are named Flat Routing (Data driven) \& various hierarchical directing (Clustering) \& Location based Routing (Geographic) routing. All these Protocols [2] are totally different from each other and they are also communicating in the network through different ways. 


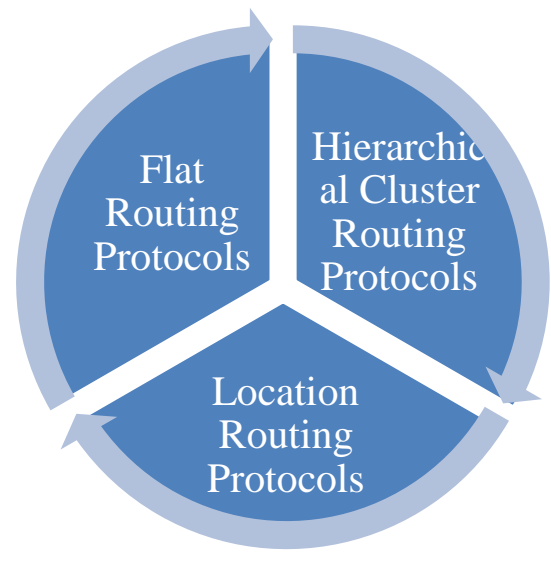

\subsubsection{Flat Routing (Data Centric)}

In Data driven directing protocols the information is transmitted to each sensor nodes which is conveyed in the arrangement district with critical excess. Each sensor hub inside Deployment district assumes the same parts like information spread and correspondence with the sink. Sink is utilized to sends request to a few locales after question sink hold up for information from the sensors that are set in the chose areas to encourage information driven attributes.

\subsubsection{Flooding and Gossiping}

The easier network routing are flooding and gossiping [3]. In Flooding each sensor nodes gets information parcel and show it to each other close-by sensor nodes which is conveying inside the area. The information arrives at the goal point and the best number of nodes is arrived at, the broadcast procedure stops. Although the fact that flooding is simple, it has no. of detriments like cover, resources visual deficiency and implosion issue. This issue is overcome by tattling. Dissimilar to traditional broadcasting which sends messages to all nearby sensors; it sends data to any neighbor. As an issue, just the certain neighbors will send the messages to the objective. Additionally it has no need of routing tables and support of topology.

\subsubsection{SPIN}

Sensor Protocol for Information via Negotiation: SPIN (Sensor Protocol for Information via Negotiation). Is versatile information driven correspondence protocol that spreads data from sensors in a vitality obliged WSN. It tackles the issue of implosion and the issue of exemplary flooding. Sensor node sending meta-information for counsel with one another before transmits the information i.e. they arrange among themselves. Arrangement is the disposal of excess information and exchange genuine data between sensor nodes. Accordingly, these nodes deal with their own particular vitality assets and decrease its abundance utilization. Turn's burden is that when exchanging the information to its target SPIN is not certain if information would reach effectively. What's more its execution is not well in the high-thickness dispersion of sensor nodes. An alternate disadvantage of SPIN is that if a few nodes are intrigued by the information that is far off from the source node and the nodes in the middle of source and target are not intrigued by the same information, then information will be fizzled conveyed to the target. Accordingly information blind sides would show up which will influence the data gathering of the system and not settling on it a decent decision for applications.

\subsubsection{Rumor Routing}

Rumor Routing is a variation of directed diffusion [3]. It routs the queries to the nodes that watches a specific event and keeps up stand out way from source to sink. On the recognition of an event, it is added to a neighborhood table called event's table. Here, it produces a perpetual packet called agent. The spread of data about nearby events to inaccessible nodes is carried out by agents. At that point they went by nodes tables are joined with their event table on the way. At whatever point an agent crosses a way which prompts an alternate event, an aggregation path is made. On the off chance that a shorter way is found by an agent than it redesigns the shorter way instantly i.e. by reviewing the essential event table. It is valuable for applications that have little number of events as the communication cost is also decreased. Additionally it helps in abstaining from flooding in SPIN.

\subsubsection{Directed Diffusion}

Directed Diffusion is an important milestone in the datacentric routing research of sensor networks [3]. The thought goes for diffusing information through sensor nodes by utilizing a naming plan for the data. The main reason for using such a scheme is to dispose of unnecessary operations of network layer routing to save energy. Directed Diffusion proposes the use of property attribute value pairs for the data and inquiries the sensors in an on interest premise by using those pair. In order to make a query, an investment is characterized utilizing a list of attribute value pairs, for example, name of objects, interval spam and geographic area, and so forth. The interest is telecast by a sink through its neighbors. Every node getting the interest can do reserving for later use. The nodes additionally can do in-network data collection, which is displayed as a minimum Steiner tree problem. The interest in the reserves are then used to contrast they got data and the qualities in the interest. The interest section additionally contains a few gradient fields. A gradient is a reply connection to a neighbor from which the interest was gotten. It is described by the data rate, term and termination time got from the got interest's fields. Thus, by using interest and gradients, paths are built in the middle of sink and sources. A few paths can be built so that one of them is chosen by reinforcement. The sink resends the first interest message through the chose path with a little interval hence reinforce the source node on that path to send data all the more as often as possible.

\subsubsection{Fermat Point Based Energy Efficient Geocast Routing}

Geocast routing protocol is utilized to exchange the packets to a gathering of nodes that are inside a specific geographical area. In decreasing the energy utilization of WASN, Fermat point based protocols in a multi-sink multi-hop are utilized which impressively decreases the aggregate transmission separation. Congested environment in WASN may expand the multipath proliferation which thusly may prompt multipath blurring. The impacts of both the elements are considered in on the execution of I-Min directing protocol intended for WASNs. I-MIN is an energy proficient plan in light of the fact that the node with higher residual energy is chosen regardless of the fact that the separation from objective is more than that for an alternate node with a less estimation of remaining energy. Radio model changes with proliferation ecological changes may influence the multipath blurring and energy utilization in a geocast routing protocol. As the quantity of Geocast regions increases, the total distance a data packet travels also increases. In this manner, the bigger is the impact 
of engendering environment when joined with the impact of multipath fading on the performance of an energy aware algorithm.

\subsubsection{Gradient-Based Routing}

Gradient Based Routing (GBR) is a reserved local predictive standard where every node figures the height of the node. It is a reserved protocol as it assigns a local threshold value to every node. In the event that the energy of an asset drops below the threshold, the height is expanded so that sensors don't send data. Although an effect on delay is defined, yet it conserves the aggregate communication energy by adjusting the dissemination of network traffic. The contrast between the node's height and its neighbor is called link gradient. Thus algorithm makes an improvement to DD [2], to get the total minimum hop numbers. Traditionally hop count was the only metric considered however now remaining energy is additionally utilized for every node while handing-off data from source to sink. This plan is helpful in a network with continuous topology change happening because of node disappointments.

\subsubsection{Two-Tier Data Dissemination}

The two-tier data dissemination (TTDD) is data driven protocol which underpins mobile sink nodes. The sensors set up a network system topology where the event message is sent to the framework. Mobile sink nodes sends request to the event related which is broadcast in the neighborhood. Accordingly it can structure a full path from the sink node to the event range. The thickness of node ought to be high. The TTDD is relying upon the transparent transmission which rearranges the support and administration of the network. TTDD take after a particular path mode in correlation to DD and reduces the data traffic Thereby, changing the network lifetime [2]. Anyhow, keeping up the grid network and worth of processing is substantial.

\subsubsection{Hierarchical Cluster Routing Protocols}

To reduce the load in carrying out long haul communication, network clustering has been used in some routing techniques which have belonged to the huge coverage area without degenerate the service. Hierarchical routing mostly process in two stages. In the first stage chose the cluster head and in the second stage routing is done. These clusters are performed data aggregation and fusion tasks to create WSNs more scalable and energy efficient.

\subsubsection{Low-Energy Adaptive Clustering Hierarchy (LEACH)}

Low-Energy Adaptive Clustering Hierarchy (LEACH) is the first hierarchical based protocol that uses randomized revolution of nearby cluster base stations (BS).LEACH is utilized when a node as a part of the network fails or the battery quits working. It is a self-organized and a versatile clustering protocol where nodes are isolated into clusters and each one cluster comprises of Cluster Head $(\mathrm{CH})$ and a Cluster Member (CM). The CHS are not chosen statically in a network as the sensor nodes may die quickly. LEACH uses randomized protocol to adjust the energy utilization for the nodes by partitioning the Cluster heads role to different nodes. Moreover, to manage the channels in a cluster LEACH utilizes TDMA (time Division Multiple Access) protocol. CHs have the obligation to allocate TDMA slots to the cluster parts. $\mathrm{CH}$ and $\mathrm{CM}$ convey shared amid the time opening that has been given to that part while different parts are in a sleep state bringing about a diminishing in energy scattering.

\subsubsection{PEGASIS and Hierarchical-PEGASIS}

Chain based protocol is a Power-Efficient Gathering in Sensor Information Systems (PEGASIS) structure chains like clusters in LEACH clustering by utilizing greedy algorithm. On account of node failed, chain is recreated such that it stays away from the dead node. In this protocol every node communicates to the closest neighbor and turn by turn transmit information to the base station. These results are less measure of energy used for every round. Data is accumulated in each round where every node gets data from one neighbor, aggregates it and transmits to an alternate neighbor in the chain. In examination to LEACH, PEGASIS performs better as it dispenses with the dynamic cluster overhead however because of asynchronous transmission time is drawn out. So it is not utilized for real time application environment. PEGASIS is changed by permitting simultaneous transmissions to happen when the nodes are not adjoining. This is known as Hierarchical-PEGASIS [3]. Despite the fact that these two algorithms wipe out the overhead of clusters, however they don't consider the energy of next hop while picking a directing path .So they are not using for heavyoverloading network [3]. Because of huge delay in information transmission it is not suitable for network where worldwide knowledge is not accessible.

\subsubsection{TEEN}

TEEN [2, 3, 4] is based on hierarchical clustering protocol, which aggregates sensors into clusters with each one headed by a $\mathrm{CH}$. The sensors inside a cluster report their sensed information to their $\mathrm{CH}$. The $\mathrm{CH}$ sends aggregated data to higher level $\mathrm{CH}$ until the information achieves the sink. Consequently, the sensor network structural planning in TEEN is focused around a hierarchical gathering where closer nodes form clusters and this methodology goes on the second level until the BS (sink) is arrived at. TEEN is for helpful for applications where the clients can control an exchange off between energy proficiency, data precision, and reaction time powerfully. TEEN utilizes a data driven technique with hierarchical methodology. Famous features of TEEN incorporate its suitability for time discriminating sensing applications. Likewise, since message transmission expends more energy than data sensing, so the energy utilization in this plan is less of what the proactive networks. Nonetheless, TEEN is not suitable for sensing applications where periodic reports are required since the client may not get any data at all if the thresholds are not arrived at.

\subsubsection{Adaptive Periodic Threshold Sensitive Energy Efficient Sensor Network Protocol (APTEEN)}

APTEEN is a change to TEEN to conquer its weaknesses and goes for both catching periodic data accumulations (LEACH) and responding to time-basic events (TEEN). Therefore, APTEEN is a cross hybrid clustering based routing protocol that permits the sensor to send their sensed information periodically and respond to any sudden change in the value of the sensed quality by reporting the relating qualities to their CHs. The construction modeling of APTEEN is same as in TEEN, which utilizes the idea hierarchical clustering for energy proficient communication between source sensors and the sink. APTEEN helps three diverse inquiry types to be specific (i) historical query, to dissect past data values, (ii) one-time inquiry, to take a preview perspective of the network; and (iii) diligent inquiry, to screen an event for a time of time. APTEEN assurances lower energy dissemination and a bigger number of sensors alive. 


\subsubsection{Hierarchical Energy Efficient Routing (HEERP)}

Protocol Hierarchical Energy Efficient Routing Protocol (HEERP) for WSN acquaints another incorporated methodology with hierarchy formation. It doesn't consider the cluster creation and cluster head choice. The algorithm mostly includes network hierarchy, neighbor table creation and information transmission. Here, the sink node launches the structuring of hierarchy by broadcasting LCREQ packet. By the meantime, a node left selects LCREQ packets from nodes with less hop numbers. Thus avoiding flooding of packets until the development of the network. In data transmission stage, every nodes sends information to their guardian nodes. Nodes failure or battery depletion like elements is considered in the maintenance stage. HEERP consume less energy than the LEACH Protocol.

\subsubsection{Location Routing (Geographic)}

Almost the routing protocols for sensor networks have need of location information for sensor nodes. In most situations location information is require in order to measure the distance between two particular nodes so that energy consumption can be evaluated. Since, there is no addressing scheme for [3] sensor networks like IP-addresses and they are deployed on a region, location information can be using in routing data in an energy efficient way.

\subsubsection{Minimum Energy Communication} Network and Small MECN (MECN and SMECN) Minimum Energy Communication Network (MECN) uses low power GPS devices and sensor nodes to set up and keep up least energy network. It finds the most diminutive network with least nodes which needs less transmission power for any two nodes (shortest path). The master- node is expected as the data sink that creates a minimum power topology for each node. Transfer area is recognized for every node which helps in more energy proficiency than with immediate transmission. With the assistance of GPS devices ideal.

\subsubsection{GEAR: Geographic Energy Aware Routing} Geographic Energy Aware Routing (GEAR) utilizes energy aware heuristics and geographic data for neighbor choice which route a packet towards the target area. It goes for decreasing the quantity of interest utilized as a part of DD by adding geographic data to the interest packet. GEAR considers a specific region to send the interest to the entire network by utilizing flooding technique. Along this way energy utilization is adjusted and network lifetime is expanded. If the destination node is close it utilizes a next hop way and in far away nodes a gap happens in GEAR. Here it advances the packet by selecting a next-hop node which minimizes the cost estimation of the neighbor. Recursive Geographic Forwarding (RGEAR) algorithm scatters the parcel inside a region. In correlation to GPSR (non-energy aware routing protocol) GEAR carries on well in uneven movement conditions.

\subsubsection{GAF and HGAF}

Geographic Adaptive Fidelity and Hierarchical Geographical Adaptive Fidelity: Geographic adaptive fidelity (GAF) [2] is an energy-aware location-based routing algorithm outlined basically for mobile ad hoc - unnecessary nodes in the system without influencing the level of routing fidelity. It structures a virtual grid for the secured region. Every node uses its GPSshown area to associate itself with a point in the virtual grid.
Nodes connected with the same point on the grid are viewed as comparable as far as the cost of packet routing. Such equality is exploited in keeping a few nodes located in a specific grid area in resting state so as to save energy. Along thus lines, GAF can considerably expand the network lifetime as the quantity of nodes increments.

2.1.3.4 GPSR: Greedy Perimeter Stateless Routing Greedy Perimeter Stateless Routing (GPSR) is a geographic routing protocol where nodes forward packet provincially in understanding greedy algorithm. It performs well in ordinary circumstances. In any case with the deterrent presentation or lacking sensors it may cause voids in network topology. GPSR illuminates this by consolidating a perimeter routing component. The nodes surrounding the voids help in their identification. In spite of the fact that this methodology functions admirably, other robust perimeter routing algorithm is additionally proposed. The chart drawn from complete network topology firstly diminishes to a planar diagram (where no edges cross). At that point the packet arriving at a void forwards the node which thusly spots the substance of planar graph. Here a node by and generally forwards the packet to the nodes with the edge that fringes the face.

\subsubsection{TBF: Trajectory Based Forwarding}

Trajectory Based Forwarding (TBF) permits packets to accept a source-specified trajectory [2]. It is a useful for direction based routing in wireless sensor networks. GPSR broadcast a packet towards the destination in a straight path, but in the case of TBF, It is uses trajectory strategy. It can also helpful in increase the efficiency of different forwarding protocols which involves the multipath forwarding with the help of spoke broadcasting and it can also broadcasting to a remote sub region. So this is very helpful in increasing the overall flexibility.

\section{CONCLUSION}

In the current days the WSNs have highly increased playing an important role for the data efficient selection and their delivery. The important issue is energy efficiency for the networks especially for WSNs which are identify by limited battery capabilities. The complexity and reliance of corporate operations on WSNs require the use of energy-efficient routing techniques and protocols, which will guarantee the network connectivity and routing of information with the less required energy [6]. In this paper, we focused on the energy efficient protocols that have been developed for WSNs.

We have mainly classified the Network structure based Routing Protocols which is the type of Routing protocols. The network [2] Structure based routing protocols further divided into three forms Flat Routing (Data driven) \& various hierarchical directing (Clustering) \& Location based Routing (Geographic) routing. Each protocol has further types of protocols that are mainly used for routing the way to the packets broadcasting from sensor to the base station. We have mainly described the energy efficiency of the protocols that is used at the time of broadcasting the packets form sensor node to the base station. In future research, concentrate on sensor network routing protocols and its integration with wired networks would be considered. For security and environmental monitoring an application is used that is mostly requires data collection from the sensor nodes to be transmitted to a server so that further analysis can be done. As the routing requirements of every environment are different, more research can be carried out considering such situations. 


\section{ACKNOWLEDGMENTS}

The authors wish to thank the faculty from the computer science department at CTIEMT, Jalandhar for their continued support and feedback.

\section{REFERENCES}

[1] Jennifer Yick, Biswanath Mukherjee, Dipak Ghosal“" On the Wireless sensor network survey" ELSEVIER, Computer Networks 52 (2008) 2292-2330

[2] Parul Khurana, Inderdeep Aulakh Punjab University Chandigarh, India "Wireless Sensor Network Routing Protocols: A Survey" International Journal of Computer Applications Volume 75- No.15, August 2013.

[3] Kemal Akkaya, Mohamed Younis "A survey on routing protocols for wireless sensor networks" ELSEVIER,

[4] M.Shanthi Dr. E.RamaDevi "A Cluster Based Routing Protocol in Wireless Sensor Network for Energy Consumption" Int. J. Advanced Networking and Applications Volume: 05, Issue: 04, Pages:2015-2020 (2014) ISSN : 0975-0290

[5] I.F. Akyildiz, W. Su*, Y. Sankarasubramaniam, E. Cayirci "Wireless sensors networks: a survey" ELSEVIER, Computer Networks 38 (2002) 393-422

[6] Nikolaos A. Pantazis, Stefanos A. Nikolidakis and Dimitrios D.Vergados, Senior Member, IEEE "EnergyEfficient Routing Protocols in Wireless Sensor Networks: A Survey" IEEE communications survey \& tutorials, vol. 15, no. 2, second quarter 2013

[7] S.K. Singh, M.P. Singh, and D.K. Singh "Routing Protocols in Wireless Sensor Networks -A Survey" International Journal of Computer Science \& Engineering Survey (IJCSES) Vol.1, No.2, November 2010m DOI:
[8] Sanjeev Kumar Gupta, Neeraj Jain, Poonam Sinha “ Clustering Protocols in Wireless Sensor Networks: A Survey" International Journal of Applied Information Systems (IJAIS) - ISSN : 2249-0868 Foundation of Computer Science FCS, New York, USA Volume 5No.2, January 2013

[9] DaWei Xu, Jing Gao,a "Comparison Study to Hierarchical Routing Protocols in Wireless Sensor Networks" ELSEVIER Procedia Environmental Sciences 10 ( 2011 ) $595-600$

[10] B. Manzoor, N. Javaid, O. Rehman, M. Akbar, Q. Nadeem, A. Iqbal, M. Ishfaq" Q-LEACH: A New Routing Protocol for WSNs " Procedia Computer Science 19 ( 2013 ) 926 - 931

[11] S. Misra et al. (eds.), Guide to Wireless Sensor Networks, Computer Communications and Networks, DOI: 10.1007/978-1-84882-218-4 4, Springer-Verlag cAst2weszvxvx London Limited 2009.

[12] Ming Zhang, Yanhong Lu, Chenglong Gong, “ EnergyEfficient Routing Protocol based on Clustering and Least Spanning Tree in Wireless Sensor Networks", International Conference on Computer Science and Software Engineering,IEEE, 2008.

[13] Geetha. V., Pranesh.V. Kallapur, Sushma Tellajeera," Clustering in Wireless Sensor Networks: Performance Comparison of LEACH \& LEACH-C Protocols Using NS2" ELSEVIER Procedia Technology 4 ( 2012 ) 163 170

[14] F. Xiangning, S. Yulin. "Improvement on LEACH Protocol of Wireless Sensor Network", 2007, International Conference on Sensor Technologies and Applications 260-264, ido:10.1109/SENSORCOMM.2007.60 